Rapid Reviews COVID-19•

\title{
Review 1: "Modelling the effectiveness and social costs of daily lateral flow antigen tests versus quarantine in preventing onward transmission of COVID-19 from traced contacts"
}

\section{Corey Peak ${ }^{1}$}

1"Bill \& Melinda Gates Foundation Global Development UNITED STATES"

Published on: Nov 10, 2021

License: Creative Commons Attribution 4.0 International License (CC-BY 4.0). 


\section{$\underline{\text { RR:C19 Evidence Scale rating by reviewer: }}$}

- Strong. The main study claims are very well-justified by the data and analytic methods used. There is little room for doubt that the study produced has very similar results and conclusions as compared with the hypothetical ideal study. The study's main claims should be considered conclusive and actionable without reservation.

\section{$* * * * * * * * * * * * * * * * * * * * * * * * * * * * * * * * * * * * * * *$}

\section{Review:}

- Is suboptimal testing independently applied for each sampling event, or do certain individuals have a $25 \%$ lower sampling ability for each sample they take on themselves? For example, I would imagine that independently applying the $25 \%$ reduction would have a smaller effect on the impact of the intervention than the assumption that certain individuals swab less deeply. I would recommend clarifying the methods and potentially including the discussion and sensitivity analysis.

- The paragraph describing Figure 2 reads "This shows that the cumulative sensitivity of repeated lateral flow testing is comparable to the one of a single clinically administered PCR test". However, Figure 2 shows the sensitivity of LFA tests. Can a line be added for PCR test sensitivity? I admit I initially thought that the green dots were PCR after reading the quoted sentence and then looking at the figure and seeing "clinical, one-off" as the legend. In addition, the authors could replicate Figure 2 but for specificity instead. There is little discussion on the concerns of false positivity.

- The end of page six reads, "However, since adherence to quarantine is uncertain and may decrease due to vaccinations and relaxation of COVID-19 related measures, we explore a wide range of adherence from 30\% to $90 \%$ ". Does this 30-90\% apply just to adherence, or the product of adherence and the percent reduction in contact rates among contacts who adhere (60\%)? If it is the former, then is the range for the overall reduction in transmission during quarantine $(0.3 * 0.6=0.18)$ to $(0.9 * 0.6=0.54)$ ? A reader may be interested in comparing DCT to a more optimistic estimate of quarantine impact since they may think that the upper bound is $90 \%$ overall when really it is $54 \%$.

- Is the socioeconomic cost of quarantine only until symptom onset (at which point, an individual would enter isolation)? Is there an assumption that individuals experiencing symptoms will isolate regardless of their intervention (DCT or quarantine)? 
- Why would individuals under DCT be spending any days in quarantine? It is unclear what the social or economic cost metric is and how it applies to both quarantine and DCT.

- Lastly, the y-axis label for the lower panels of Figure 3 is not clear for the DCT condition. Further, in Figure 3, each line appears to flatten at day 13. I gather that this is the day quarantine would end $(3+10)$, but why do the DCT lines also flatline at day $10(3+7)$ ? 